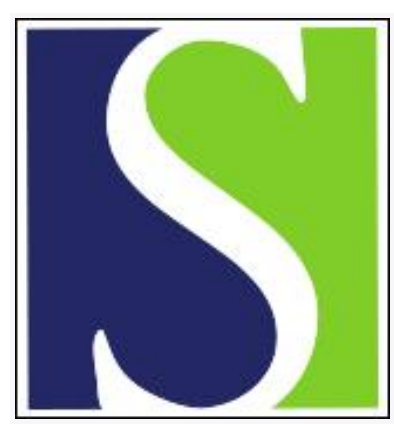

Scand J Work Environ Health 1998;24(1):1-2

https://doi.org/10.5271/sjweh.270

Issue date: Feb 1998

Cancer registries in environmental cancer epidemiology by Teppo L

This article in PubMed: www.ncbi.nlm.nih.gov/pubmed/9562393

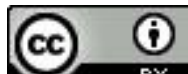




\section{Cancer registries in environmental cancer epidemiology}

Environmental exposures occupy an important position in cancer causation. Even if the etiologic fractions associated with various life-style factors such as smoking, diet, alcohol consumption, and sexual habits may, according to current knowledge, be greater than those of exposures occurring in the human environment, it is often easier to change the environment than to make individuals change their life-style and habits into healthier modes. Through legislation and various technical measures it is often possible to regulate the occurrence of different carcinogenic agents in the human environment and to diminish exposures to them as has been done with, for example, carcinogens at workplaces, ionizing radiation, and ambient air and water pollution.

Before regulatory measures can be undertaken, the individual exposures and their relative importance should be known. Useful information is obtained through laboratory research, but its relevance in the human setting should, when possible, be confirmed on the population level, that is, by using epidemiologic methods. Such questions as how high are the relative risks associated with exposures that occur in real life, how long are the latencies, and are there important interactions need to be answered.

In countries and areas where a well-functioning cancer registry exists, epidemiologic cancer research is greatly facilitated. If a cohort defined by exposure to a suspected agent can be created, cases of cancer can be identified from the registry files by applying usual cohort study methodology. Cancer registry may also be able to produce unbiased case series for case-referent studies, and these data are especially important when rare cancers are concerned. (The number of cases would be too small in any cohort study.) Quite often, a case-referent approach within a cohort is used.

In each Nordic country there is a cancer registry covering the entire population of the country, and the individual person numbers available in the countries make the record linkage between study cohorts and registry files a technically easy procedure that has been successfully used in a large number of epidemiologic studies on both the national and Nordic level. Confidentiality rules concerning the registries are reasonable and have not caused undue problems for appropriate epidemiologic studies.

The Nordic cancer registries have participated in several studies in which environmental exposures have been analyzed in terms of cancer risks. In Finland, both cohort studies and case-referent studies have been conducted, recently focusing, for example, on low-intensity magnetic fields produced by high-voltage powerlines (only a marginal increase in the risk of adult leukemia found), chlorophenols in drinking water after leakage from a sawmill (risk of non-Hodgkin's lymphoma slightly increased), and ionizing radiation caused by radon daughters from the earth (suggestive increase in the risk of lung cancer) or by fallout from the Chernobyl accident (no increase in the risk of childhood leukemia).

Further studies in which data from cancer registries have been successfully utilized include an evaluation of the cancer risks associated with several exposures in the work environment, the risk of leukemia among people who live close to an oil refinery, the risk of cancer associated with the mutagenicity of drinking water due to chlorination procedures, and the risk of sarcoma and other cancers among herbicide users.

In this issue of the Scandinavian Journal of Work, Environment \& Health, a review article is published on environmental risk factors in breast cancer by Esther A Welp and her co-workers. In their conclusions, the authors end with several proposals for future studies. These proposals are well 
focused. Breast cancer is one of the most common cancers in the western world. In spite of extensive research carried out for decades in all parts of the world, simple effective preventive measures are still lacking. The strongest risk factors that have been found are related to endogenous estrogen load and the reproductive history of women, but these factors can only explain a part of all breast cancers. Moreover, even if many of these factors are well characterized, it is difficult, if not impossible, to utilize them in prevention. Thus better understanding of the environmental risk factors for breast cancer would be extremely important. Although the relative risks associated with them are probably low, a large number of women are likely to be exposed, the result being substantial numbers of preventable cancers.

One group of chemicals in the human environment that is of interest in terms of breast cancer causation is the group of so-called hormone disruptors. They are compounds with weak estrogenic effects in animal experiments or in vitro studies. Examples of these chemicals are organochlorine compounds (DDT, PCB, etc), phthalates, tributyltin compounds, and alkylphenols. They occur in pesticides, paints and plastic products, anti-fouling agents, and many other products used in a variety of ways. Many people are exposed to them in their workplaces and everyday life - often without knowing it.

An association between hormone disruptors and male infertility, cryptorchidism, and testis cancer has been suggested, and epidemiologic studies on these issues have been made. In addition the hypothesis of a link between exposure to hormone disruptors and breast cancer is biologically plausible. Data supporting this association are increasing, but problems will arise even in well-designed epidemiologic studies. Reliable data on life-long exposures to hormone disruptors are difficult to obtain, and studies based on chemical analyses of fat tissue from cases and referents (aiming at the estimation of exposures) have their limitation. Still almost everyone in western populations is exposed to them at least to some extent.

Collaboration between different areas is needed in modern cancer epidemiologic research. Solving the problem of the association between hormone disruptors and breast cancer causation calls for cooperation between epidemiologists, chemists, endocrinologists, cell biologists, and experts in gene research. And behind all these are the cancer registers with their data on cancer patients, which may be helpful in certain approaches.

\section{Lyly Teppo}

Finnish Cancer Registry, Helsinki, and School of Public Health, University of Tampere, Tampere

Address for correspondence:

Finnish Cancer Registry

Institute for statistical and Epidemiological Cancer Research

Liisankatu $21 B$

FIN-00170 Helsinki

Finland 